\title{
ARTIGOS
}

\section{HEIDEGGERS ERLÄUTERUNG DER HÖLDERLINSCHEN DICHTUNG: DIE VERWANDLUNG DES BIOGRAPHISCHEN IM GEDICHTETEN*}

\author{
Choong-Su Han** \\ http://orcid.org/0000-0003-2366-745X \\ choong-su.han@ewha.ac.kr
}

ZUSAMMENFASSUNG Die vorliegende Arbeit unternimmt den Versuch, zu erklären, was Heidegger mit dem Ausdruck "die Verwandlung des Biographischen im Gedichteten” bei seiner Erläuterung der Hölderlinschen Hymne Andenken meint. Viele Literaturwissenschaftler interessieren sich für das Entstehungsdatum der Hymne und die damaligen Erlebnisse Hölderlins, um daraus die Hymne zu interpretieren. Im Vergleich mit ihnen richtet Heidegger wenig sein Augenmerk darauf, weil er glaubt, dass das Biographische des Dichters unmittelbar nichts mit dem Inhalt des Gedichtes zu tun hat. Bei der Dichtung geschieht Heidegger zufolge die Verwandlung des Biographischen im Gedichteten. Demnach untersucht die vorliegende Arbeit das Phänomen des Dichtens. Dazu wird Heideggers Erläuterung der Hymne Wie wenn am Feiertage ... herangezogen, die er als die "reinste Dichtung des Wesens der Dichtung" ansieht. Danach wird das Gedicht Andenken im Hinblick auf die "Schiffer" betrachtet, die Heidegger die "kommenden Dichter Germaniens" nennt. Diese Betrachtung dient dazu, die Verwandlung, die im Dichten geschieht, zu erläutern.

Schlüsselwörter Heidegger; Hölderlin; Andenken; Verwandlung; Dichtung.

* Article submitted on 17/09/2018 and accepted on 30/01/2019.

** Ewha Womans University. Seoul, Republic of Korea. 
ABSTRACT Iattempt to explain what Heidegger means by the expression "the transformation of the biographical data in the poem" in his elucidation of the Hölderlin's hymn Remembrance. Many scholars of German literature are interested in the date of the hymn and in Hölderlin's experience at that date, in order to interpret the hymn. In contrast to them, Heidegger does not concern himself with the date, because he believes that the poet's biographical data has nothing to do with the content of the poem directly. According to Heidegger, the transformation of the biographical data in the poem takes place through a process of poeticizing. Therefore, I investigate this process as the nature of the poetry. For this investigation, I refer to Heidegger's elucidation of the hymn As When on Holiday ..., which he regards as the "purest poem on the essence of poetry". Afterwards, I interpret the hymn Remembrance in view of the "mariners", which Heidegger calls "Germania's coming poets". This interpretation serves to help us understand the transformation that occurs in the process of poeticizing.

Keywords Heidegger; Hölderlin; Remembrance; Transformation; Poetry.

\section{Fragestellung}

Mit Hölderlins Gedicht Andenken setzte sich Heidegger intensiv zweimal auseinander. Zum einen hielt er die Vorlesung Hölderlins Hymne "Andenken” im Wintersemester 1941/42 an der Universität Freiburg. Zum anderen schrieb er den Beitrag “Andenken” zum hundertsten Todestag Hölderlins im Jahr 1943. ${ }^{1}$

In der Vorbetrachtung der genannten Vorlesung kündigt Heidegger (1982, p. 1) eigentlich an, dass er sich mit fünf Gedichten Hölderlins beschäftigen möchte, nämlich mit Andenken, Der Ister, Die Titanen, Mnemosyne und Reif sind ... Diesem Plan entsprechend trägt die Handschrift der Vorlesung den Titel Hölderlins Hymnen. In der Notiz Aufbau der Auslegung der genannten Dichtungen, die im September 1941, also vor dem Wintersemester 1941/42, gemacht wurde, stellt Heidegger (1982, p. 197) fest: "Die Auslegung von 'Andenken' gibt den Grund und Ausblick und die Hinsichten für alles Folgende". Was daher im Zentrum der genannten Vorlesung steht, ist das Gedicht Andenken. Die vier anderen Gedichte werden dabei nur gelegentlich erwähnt. Demnach

1 Der Beitrag ist eine kompakte Darstellung der Vorlesung. Sie sind im Grunde genommen inhaltlich ähnlich. Ausweichende Stellen werden nur dann betrachtet, wenn sie relevant für das Unternehmen der vorliegende Arbeit sind. 
lautet der Titel der Vorlesung nicht Hölderlins Hymnen, sondern Hölderlins Hymne "Andenken".

Das Gedicht Andenken besteht aus fünf Strophen. Während die ersten vier Strophen jeweils 12 Verse haben, schließt die fünfte Strophe bereits mit dem elften Vers. Auf den ersten Blick hängt das Gedicht scheinbar inhaltlich mit persönlichen Erlebnissen Hölderlins zusammen, weil es Stadt ("Bourdeaux [sic]"), Flüsse ("Dordogne" und "Garonne"), Landschaft (Bäume, "Ulmwald" usw.) und Menschen ("braune Frauen") des südlichen Frankreichs beschreibt, in dem sich Hölderlin vor der Abfassung des Gedichtes aufgehalten hatte (Hölderlin, $1992^{1}$, p. 28). Dieser Zusammenhang zwischen dem persönlichen Erlebnis und dem Gedichteten interessiert Heidegger aber nicht, weil er die "Verwandlung des Biographischen im Gedichteten" behauptet (Heidegger, 1982, p. 28). Wenn das Biographische in das Gedicht verwandelt, also völlig geändert, würde, dann wäre es sinnlos, die Spur des Biographischen im Gedicht zu finden. Ein direkter Zusammenhang zwischen den beiden würde nicht bestehen.

In der vorliegenden Arbeit unternehmen wir ${ }^{2}$ den Versuch, zu erklären, was Heidegger mit der Verwandlung meint. Da dieser Begriff nur in der Vorlesung Hölderlins Hymne "Andenken" einmal vorkommt und keine nähere Erläuterung findet, müssen wir einen Umweg einschlagen, indem wir uns fragen, wie die Verwandlung des Biographischen in das Gedicht geschieht. Sie vollzieht sich wahrscheinlich beim Dichten. Demnach setzen wir uns mit dem Phänomen des Dichtens oder mit dem Wesen der Dichtung auseinander, um dadurch den Begriff der Verwandlung verständlich zu machen. Dabei berufen wir uns auf Heideggers Erläuterung der Hymne Wie wenn am Feiertage ..., ${ }^{3}$ die er als die “reinste Dichtung des Wesens der Dichtung” ansieht (Heidegger, 1981, p. 44).

Der Hauptteil der vorliegenden Arbeit besteht aus drei Paragrafen. Im ersten Paragrafen gehen wir auf die Debatte um die Frage ein, wann das Gedicht Andenken entstanden ist. Heideggers Antwort auf die Frage ist einfach: "Entstanden ist es [scil. das Gedicht Andenken] wohl um 1803/04" (Heidegger, 1981, p. 79; Heidegger, 1982, p. 19). Dafür bringt er aber keine Begründung vor. Seine einfache Antwort hängt wohl mit seiner eigenen, desinteressierten Stellungnahme zur Debatte zusammen. Um Heideggers Position deutlich zu machen, betrachten wir vier Literaturwissenschaftler, die jeweils verschiedene Datierungen hinsichtlich der Entstehung des Gedichtes vornehmen. Im zweiten

2 Der Autor der vorliegenden Arbeit verwendet nicht "ich", sondern "wir", um dadurch die Leser in seinen Gedankengang einzubeziehen (Bußmann, 1983, p. 395).

3 Diese Hymne beginnt mit folgenden Worten: "Wie wenn am Feiertage, das Feld zu sehn [...]". Aufgrund dieses ersten Verses trägt die Hymne den Titel Wie wenn am Feiertage ... Abkürzend wird sie auch Feiertagshymne genannt. 
Paragrafen widmen wir uns der Hymne Wie wenn am Feiertage ..., um das Wesen der Dichtung dort anhand der Heideggerschen Erläuterung dieser Hymne aufzuzeigen. Es ist die Begeisterung, die aber nicht im heutigen Sinne verstanden werden darf, sondern in engem Zusammenhang mit dem Geist steht. Im dritten und letzten Paragrafen des Hauptteils kommen wir zum Gedicht Andenken zurück. Wir betrachten die "Schiffer", die für Heidegger (1981, p. 86) die "kommenden Dichter Germaniens" sind, die das "Heilige" sagen. Unsere Betrachtung dient dazu, die Verwandlung, die im Dichten geschieht, zu erläutern.

\section{Der Entstehungszeitpunkt des Gedichtes Andenken}

Das Gedicht Andenken mit den beiden großen Hymnen Patmos und Der Rhein erschien in der Zeitschrift Musenalmanach im Jahr 1808; was die Zeit der Niederschrift des Gedichtes betrifft, ist sie aber sehr umstritten (Baumann, 1997, p. 11). Friedrich Beißner vermutet, dass das Gedicht Andenken "im Frühjahr 1803, bald nach der Vollendung der ersten Fassung des PatmosGesangs, entstanden" ist. ${ }^{4}$ Jochen Schmidt ist der gleichen Meinung: "Von dieser nicht sicher datierbaren, wahrscheinlich im Jahr 1803 entstandenen Hymne [Andenken] ist nur die Schlußstrophe handschriftlich überliefert." Hingegen behauptet Dietrich Sattler (1981, p. 609), dass das Gedicht erst nach dem März 1805 geschrieben wurde, und zwar deswegen, weil er "ein edel Paar/von Eichen und Silberpappeln" als Trauerbäume versteht, die an den Tod von Friedrich Klopstock (1724-1803) und den von Wilhelm Heinse (1746-1803) erinnern. Roland Reuß (1990, p. 101) hält die Datierungsversuche Beißners und Sattlers insofern für gescheitert, als die Gleichheit zwischen dem Autor des Gedichtes und dem poetischen Ich im Gedicht nicht garantiert ist. Außerdem zweifelt Reuß daran, dass das Gedicht Andenken im Frühjahr oder im März entstanden ist; denn Hölderlin hat das Gedicht einige Zeit nach dem Frühlingsäquinoktium, das im Gedicht genannt wird, zu Papier bringen können; nur Folgendes ist festzuhalten, "daß Hölderlin das Gedicht nach dem Aufenthalt in Bordeaux, d. h. dem Frühjahr 1802, und vor seiner Einlieferung ins Autenriethsche Klinikum (in jenem denkwürdigen Herbst des Jahres 1806) geschrieben haben muß” (Reuß, 1990, p. 101). Stuttgarter Ausgabe, Band 2), Stuttgart: Cotta, 1990.

5 Schmidt ist der Herausgeber der Deutschen Klassiker Ausgabe. Vgl. Friedrich Hölderlin, "Überblickskommentar", in: Sämtliche Gedichte, Frankfurt am Main: Deutscher Klassiker Verlag, 1992. 
Die vier genannten Literaturwissenschaftler beschäftigen sich damit, den Entstehungszeitpunkt des Gedichtes mehr oder weniger festzulegen. Anders als sie interessiert sich Heidegger kaum für die genaue Datierung. Sein Desinteresse liegt daran, dass das Gedicht Andenken "seinen Wesensursprung nicht in dem berichteten Aufenthalt in Frankreich" hat (Heidegger, 1981, p. 83). Vielmehr basiert das Gedicht auf der erwähnten Verwandlung. Es ist aber nicht klar, in welcher Weise sich die Verwandlung vollzog und wie lange sie dauerte. Daher ist der Entstehungszeitpunkt des Gedichtes nicht fixierbar. Also tritt Heidegger in die Debatte um die Frage nach dem Entstehungszeitpunkt des Gedichtes Andenken überhaupt nicht ein.

Die Bücher der genannten Literaturwissenschaftler sind nach dem Tod Heideggers erschienen. Mit ihnen hat er sich eigentlich nicht auseinandergesetzt. Als Heidegger die Verwandlung des Biographischen im Gedichteten behauptete, war sein Gegenspieler Norbert von Hellingrath, der den Dichter Hölderlin im zwanzigsten Jahrhundert neu entdeckt hat. Von Hellingrath meint, dass das Gedicht Andenken "persönliche Erlebnisse des Menschen Hölderlin", "die Erinnerung an die Landschaft von Bordeaux, an die Freunde dort, die sich auf grosse Seefahrt wagen, und die Gefühle[,] die dieses Gedenken in ihm weckt," "zum Gegenstand" hat. ${ }^{6}$ Aufgrund dieser Meinung ordnet von Hellingrath dieses Gedicht unter die lyrischen Gedichte ein. Dagegen erhebt Heidegger (1982, p. 24) folgende Einwände, 'ob dieses Gedicht 'Andenken' nur an persönliche Erlebnisse erinnert, ob es überhaupt persönliche Erlebnisse meint, ob 'Andenken' hier nur soviel besagt wie Erinnerung an Vergangenes."

Auf diese Fragen antwortet Heidegger, indem er sich mit der Dunkelheit des Gedichtes Andenken auseinandersetzt. Zum Beispiel weist er auf den rätselhaften Bezug zwischen dem Titel Andenken und dem Schlussvers des Gedichtes ("Was bleibet aber, stiften die Dichter.") hin. Das Wort "stiften" ist hier bemerkenswert, weil das Stiften nicht mit dem "Gedächtnis an Vergangenes", sondern mit der "Gründung eines Künftigen" zusammenhängt. (Heidegger, 1982, p. 24). Mit diesem Beispiel konstatiert Heidegger, dass das "Andenken" nichts mit der Erinnerung an Vergangenes zu tun hat. Auch im Hinblick auf die Dunkelheit des Gedichtes Andenken nimmt Heidegger (1982, p. 43) die "psychologisch-biologische Erklärung" nicht an, der zufolge Hölderlins Dichtung aus dem Zeitraum zwischen 1800 und 1806 das "'Produkt' eines 'Verrückten"”

6 Von Hellingrath ist der Herausgeber der folgenden Ausgabe: Friedrich Hölderlin, Gedichte 1800-1806 (Hölderlins Sämtliche Werke, Band 4), München/Leipzig: Müller, 1916. Vgl. Martin Heidegger, Hölderlins Hymne "Andenken", Frankfurt am Main: Vittorio Klostermann, 1982, p. 23. 
ist. ${ }^{7}$ Heidegger distanziert sich von der "psychologisch-biographischen Erklärungssucht”, weil er (1982, p. 46) meint: “Alles Biologische-Psychologische hilft uns nichts zur Erläuterung der Gedichte, weil das Biographische umgekehrt erst aus dem Werk seine Deutung und Bestimmung erfahren kann." Diese Umkehrung hat etwas mit der Verwandlung zu tun, in der das Wesen der Dichtung besteht. Nunmehr ziehen wir die Hymne Wie wenn am Feiertage ... heran, um das Wesen der Dichtung im Hinblick auf die Begeisterung zu verstehen.

\section{Das Wesen der Dichtung als Begeisterung}

Die Feiertagshymne beginnt damit, den morgendlichen Spaziergang eines Landmannes zu beschreiben. Dabei zeigt sich, dass die Naturdinge (Strom, Boden, Weinstock und Bäume) nach dem nächtlichen Gewitter frisch erscheinen. So wie sie "unter günstiger Witterung" stehen, sind die Dichter durch die "mächtige" und "göttlichschöne Natur" "in leichtem Umfangen" erzogen (Hölderlin, 1992 ${ }^{1}$, p. 239). Die Dichter bringen die Natur, die Hölderlin in das Heilige in der dritten Strophe der Hymne umbenennt, zur Sprache. Die heilige Natur - so wie der vernichtende Blitzstrahl - ist sehr gefährlich, sodass die Erdensöhne entzündet und verbrannt werden, wenn diese sie unmittelbar erfahren. Aber durch die Dichtung der Dichter erfahren, oder mit Hölderlin (1992 ${ }^{1}$, p. 240) gesagt, "trinken" die Erdensöhne "himmliches Feuer" "ohne Gefahr".

Das Wort "Begeisterung" findet sich in der dritten Strophe der Feiertagshymne: “[...] Fühlt neu die Begeisterung sich,/Die Allerschaffende wieder" (Hölderlin, $1992^{1}$, p. 239) Mit der Begeisterung, die alles erschafft, setzt Hölderlin die Natur gleich. Diese Gleichsetzung erweckt einen merkwürdigen Eindruck, weil ein innerlicher Zustand des Menschen und die Natur außerhalb des menschlichen Körpers miteinander identisch sind. Aus diesem Eindruck stellt sich die Frage, ob Hölderlin das Wort "Begeisterung" im anderen Sinne versteht als die Heutigen.

Um darauf zu antworten, betrachten wir zunächst drei wichtige Deutungen der Feiertagshymne, die zahlreiche Interpretationen hervorgerufen hat: die "gründlichste Analyse" (Seifert, 1982, p. 93) von Peter Szondi, die jüngste Monografie von Jürg Friedrich und schließlich den Kommentar von Jochen Schmidt, der die Hölderlin-Ausgabe Sämtliche Werke und Briefe im Jahr 1992 herausgab. Danach ziehen wir Heideggers Erläuterung der Feiertagshymne heran,

7 Vgl. Martin Heidegger, Hölderlins Hymne “Andenken”, Frankfurt am Main: Vittorio Klostermann, 1982, p. 43: "Über die alles hinaus und diesem allem zuvor, was da an 'Inhalt' des Gedichtes angeführt werden könnte, besteht wohl ein Zusammenhang, der zunächst verborgen bleibt. Schon die innere Verklammerung der fünf Strophen ist dunkel." 
in welcher sich eine klare Antwort auf die obengenannte Frage findet: "Die Natur be-geistert alles als die allgegenwärtige, allerschaffende. Sie ist selbst die 'Begeisterung'. Be-geistern kann sie nur, weil sie 'der Geist' ist' (Heidegger, 1981, p. 60). Die Betrachtung der genannten Deutungen ist dafür tauglich, die Eigentümlichkeit der Heideggerschen Erläuterung der Feiertagshymne deutlich zu machen, welche sogar als seine eigenen Gedanken aufgefasst werden kann, die er in die Feiertagshymne hineinprojiziert hat.

In seinem Buch Einführung in die literarische Hermeneutik richtet Peter Szondi sein Augenmerk nicht auf das Wort "Begeisterung". Er betrachtet vielmehr nur das Wort "Geist", das in den letzten zwei Versen der fünften Strophe der Feiertagshymne vorkommt: "Des gemeinsamen Geistes Gedanken sind,/Still endend in der Seele des Dichters" (Hölderlin, $1992^{1}$, p. 240). Szondi (1975, p. 275) bestimmt aber nicht, was dieser Geist bedeutet. Jürg Friedrich zufolge ist die allerschaffende Begeisterung allem Entstehen gemeinsam. Sie wohnt also nicht nur allem natürlichen Werden, sondern auch allem menschlichen Schaffen inne. Die Begeisterung ist die "hervorbringende Kraft", die die Dichter und die Natur gemeinsam haben (Friedrich, 2007, p. 91). Jochen Schmidt zufolge stellt die Feiertagshymne den Übergangsprozess vom unbewussten zum geistigen Zustand im Hinblick auf die Natur und die Dichter dar. Die Übergangsmomente bringt Hölderlin "in deutlich kompositorischer Absicht" immer im jeweils vorletzten Vers der verschiedenen Strophen zur Sprache. (Hölderlin, 1992¹, p. 657). Die Natur, die in der zweiten Strophe zu schlafen scheint und ahnend ruht, erwacht aus dem Schlaf in der dritten Strophe und fühlt sich wie neugeboren, deswegen befindet sie sich nun im Zustand des Sich-Fühlens. In der vierten Strophe ist die Rede von der Erkenntnis ${ }^{8}$ der Natur, die in der fünften Strophe vergeistigt wird. So steigert sich die Natur zum Geist. Da die Begeisterung als "gefühlshafte Vorstufe des Geistes" verstanden wird, hat sie noch keinen Geist und ist noch kein Geist (Hölderlin, 1992¹, p. 658). Während Szondi das Wort "Begeisterung" gar nicht beachtet, gehen Friedrich und Schmidt darauf ein, aber sie identifizieren die Begeisterung und die Natur nicht miteinander. Für Friedrich ist die Begeisterung die Kraft der Natur. Schmidt sieht die Begeisterung als das Zwischenstadium im Verlauf der Entwicklung der Natur zum Geist an.

8 Im Hinblick auf die vierte Strophe der Feiertagshymne gibt es einige Unterschiede zwischen den Ausgaben Norbert von Hellingraths und Jochen Schmidts. Ein wichtiger Unterschied besteht in den letzten zwei Versen. Der ersten Ausgabe zufolge lauten sie: "In Knechtsgestalt, sie sind bekannt, die/Allebendigen, die Kräfte der Götter". Hingegen lauten die Verse in der zweiten Ausgabe: "In Knechtsgestalt, sie sind erkannt,/Die Allebendigen, die Kräfte der Götter". Die Wörter "bekannt" und "erkannt" sind voneinander zu unterscheiden. Diesen Unterschied formuliert Hegel (1970, p. 35) mit folgenden Worten: "Das Bekannte überhaupt ist darum, weil es bekannt ist, nicht erkannt." Heidegger zitiert die Ausgabe von Hellingraths, weswegen er nicht über die Erkenntnis in seiner Erläuterung der Feiertagshymne spricht. 
Heidegger setzt, wie gesagt, die Natur, die Begeisterung und den Geist miteinander gleich. Dabei ist bemerkenswert, dass er das Wort "Begeisterung" in zwei Wortteile spalt. Diese Spaltung findet sich erstmals in folgendem Satz: "[A] lles Erscheinende [ist] vom Geist durchstrahlt: be-geistert" (Heidegger, 1981, p. 60). Heideggers Bestimmung der Begeisterung als Durchstrahlung des Geistes ist aber ungewöhnlich, weil sie nicht der heutigen Definition der Begeisterung als der "freudigen Erregung" entspricht. ${ }^{9}$ Wenn wir aber berücksichtigen, dass das Wort "begeistern" ursprünglich "mit Geist erfüllen" bedeutete, ${ }^{10}$ dann erkennen wir einen engen Zusammenhang zwischen "begeistern" und "Geist". Um das Wort "begeistern" im ursprünglichen Sinne zu verwenden, hebt Heidegger das darin geborgene Wort "Geist" durch den Bindestrich hervor. Im Hinblick auf die Begeisterung in ihrem ursprünglichen Sinne und in ihrem Zusammenhang mit der Natur und dem Geist betrachten wir nun die Hymne Wie wenn am Feiertage ...

Im Mittelpunkt der Feiertagshymne steht die Natur: "Was Hölderlin hier [scil. in der zweiten Strophe] noch 'Natur' nennt, durchstimmt das ganze Gedicht bis in sein letztes Wort” (Heidegger, 1981, p. 52). In der heiligen Natur erscheint und leuchtet alles Seiende, das ähnlich wie eine Feuerstelle ist, die durch die "[1]ichte" Natur "befeuert" wird; durch das "[1] euchten[de]" "Erscheinen" hebt sich jedes Seiende von seiner Umgebung ab, unterscheidet sich von den anderen Seienden und steht "in seinem eigenen Umriß und Maß" (Heidegger, 1981, p. 60). Heidegger verwendet also die Metapher des Feuers. Diese Verwendung ist aber nicht seine willkürliche Entscheidung, weil das Wort "Feuer" mehrmals in der Feiertagshymne vorkommt, und zwar zweimal in der vierten Strophe und einmal in der siebten Strophe. Auch Szondi (1970, p. 41) behauptet, dass das Motiv, das die Hymne von Anfang bis Ende durchzieht, das "himmlische Feuer" ist."

Der Brennstoff, womit die feurige Natur alles Seiende versorgt, ist um bei der Feuermetapher weiter zu bleiben - nichts anderes als die Luft. Jedes Seiende atmet diese Luft ein, die in ihm verbrennt, um die Wärme zu erzeugen. So durchstrahlt die Luft jedes Seiende. Das, was alles Erscheinende durchstrahlt, nennt Heidegger aber nicht die Luft, sondern den Geist. Um die Gleichheit zwischen Luft und Geist zu verstehen, betrachten wir die

9 Das Bedeutungswörterbuch (Band 10), Ed. Dudenredaktion, Mannheim/Leipzig/Wien/Zürich: Duden, 2002, p. 184.

10 Herkunftswörterbuch. Etymologie der deutschen Sprache (Band 7), Ed. Dudenredaktion, Mannheim/Leipzig/ Wien/Zürich: Duden, 2001, p. 262.

11 Auch Derrida (1992, p. 7) versteht Heideggers Begriff des Geistes im Hinblick auf das Feuer: "Ich [scil. Derrida] werde vom wiederkehrenden Geist, von der Flamme und von der Asche sprechen." 
ursprüngliche Bedeutung des Geistes. Im Rahmen der Christianisierung wirkten das altgriechische Wort " $\pi v \varepsilon \tilde{v} \mu \alpha$ " und das lateinische Wort "spiritus" auf das deutsche Wort "Geist" ein. Das altgriechische Wort " $\pi v \varepsilon \tilde{u} \mu \alpha$ " bedeutet unter anderem der "Hauch", "Luftstrom", "Atem", die "Seele", der "Geist". ${ }^{2}$ Das lateinische Wort "spiritus" bedeutet: unter anderem der "Hauch, Lufthauch, Luftzug, die wehende, bewegte Luft", das "Einatmen der Luft, das Atemholen, der Atem", der "Geist, die Seele". ${ }^{13}$ Aufgrund der Bedeutungen von " $\pi v \varepsilon \tilde{v} \mu \alpha$ " und "spiritus" sind die Natur, die die Luft versorgt, und der Geist miteinander identisch. Die Natur als Begeisterung erfüllt jedes Seiende mit Luft und Geist. Sie begeistert das Seiende im Ganzen, weil sie selbst der Geist ist.

Es ist bemerkenswert, dass das Wort "Begeisterung" am Ende der Heideggerschen Erläuterung der Feiertagshymne wieder vorkommt: "Das Kühle und Schattige des Nüchternen entspricht dem Heiligen. Diese Nüchternheit verleugnet nicht die Begeisterung. Die Nüchternheit ist die allzeit bereite Grundstimmung der Bereitschaft für das Heilige" (Heidegger, 1981, p. 77). Also schließt Heidegger (1981, p. 76) seine Erläuterung der Feiertagshymne, die ihm zufolge nichts anderes als die "Hymne 'des' Heiligen" ist, mit dem Hinweis auf die Nüchternheit, die aber nicht im Gegensatz zur Begeisterung steht. Das innige, aber scheinbar gegensätzliche Verhältnis von Nüchternheit und Begeisterung erinnert uns an eine bekannte Stelle in der Hölderlinschen Skizze Reflexionen: "Es gibt Grade der Begeisterung. [...] Auf dieser auf- und abzusteigen ist Beruf und Wonne des Dichters. [...] Da wo die Nüchternheit dich verläßt, da ist die Grenze deiner Begeisterung. Der große Dichter ist niemals von sich selbst verlassen, er mag sich so weit über sich selbst erheben als er will. Man kann auch in die Höhe fallen, so wie in die Tiefe. Das letztere verhindert der elastische Geist, das erstere die Schwerkraft, die in nüchternem Besinnen liegt" (Hölderlin, 1992², p. 519). In dieser Skizze ist bemerkenswert, dass Hölderlin die Begeisterung nicht auf die Natur, sondern auf den Dichter bezieht. Sowohl in Hölderlins Feiertagshymne als auch in der Heideggerschen Erläuterung dieser Hymne betrifft das Wort "Begeisterung" hauptsächlich nur die heilige Natur. Aber am Schluss dieser Erläuterung sowie in Hölderlins Reflexionen gehört die nüchterne Begeisterung auch zum Dichter. ${ }^{14}$ Diese Zugehörigkeit ermöglicht unsere Auslegung der Begeisterung als Wesen der Dichtung.

12 Wilhelm Gemoll, Griechisch-Deutsches Schul- und Handwörterbuch, München/Wien: Freytag, 1988, p. 612.

13 Ausführliches Lateinisch-Deutsches Handwörterbuch (Band 2), Ed. Heinrich Georges, Hannover: Hahn, 1983, pp. 2763-2765.

14 Auch Jürg Friedrich (2007, p. 91) weist darauf hin, dass die allerschaffende Begeisterung ("hervorbringende Kraft") nicht nur zur Natur, sondern auch zur Kunst gehört. 
Wir betrachten die Feiertagshymne im Hinblick auf den Entstehungsprozess des Liedes. Offenbar besteht dieser Prozess aus den vier Stufen. Zunächst "ahnen" die Dichter die "ruhe[nde]" Natur. Danach "erwacht" die Natur als das Heilige und "fühlt" sich neu wieder; die Dichter nehmen das heilige Feuer dadurch wahr, dass es "in [den] Seelen der Dichter" "angezündet" wird. Im Anschluss daran hüllen die Dichter das "himmlische Feuer", das sie "mit eigner Hand" gefasst haben, "ins Lied". Schließlich "reichen" sie dem Volk das Lied, in dem der Geist der Natur "wehet" (Hölderlin, 1992", pp. 239-240). Diesem Entstehungsprozess (Ahnen, Wahrnehmen, Einpacken und Geben) wohnt ein durchgängiges Prinzip der Begeisterung inne, nämlich die Begeisterung der Natur und des Heiligen und die Begeisterung in den Dichtern und im Lied. In der Hymne Wie wenn am Feiertage ... lässt sich das Wesen der Dichtung daher als Begeisterung bestimmen.

Im Hinblick auf die Aufgabe der vorliegenden Arbeit fragen wir, auf welcher Stufe der Dichtung die Verwandlung geschieht. Da die Begeisterung durchgängig während des ganzen Dichtungsprozesses herrscht, scheint es, dass sich die Qualität der Begeisterung nicht verändert. Diese erfahrt jedoch aber eine große Änderung auf der dritten Stufe. Wenn das himmlische Feuer ins Lied nicht gehüllt wird, ist es für die Erdensöhne zu gefährlich, weil es im Zustande ist, sie sowie Semele einzuäschern. Die zerstörerische Kraft des feurigen Geistes stillt sich und ruht in den Seelen der Dichter. Dieser gestillte Geist wird ins Lied weitergeleitet: "[I]m Lied wehet ihr Geist" (Hölderlin, $1992^{1}$, p. 240). Aufgrund dieser Verwandlung im Sinne der Milderung der Gefahr unterscheidet sich die Erfahrung des Dichters, also die biographische Erfahrung, vom Gedichteten in seinem Gedicht.

\section{Die Begeisterung und die Verwandlung im Gedicht Andenken}

In der Feiertagshymne haben wir die Verwandlung bezüglich der Begeisterung aufgezeigt. Dementsprechend fangen wir mit dem Begriff der Begeisterung im Gedicht Andenken an, um die Verwandlung dort sichtbar zu machen. In der Vorlesung Hölderlins Hymne "Andenken” kommt das Wort "Begeisterung" zweimal vor. An der ersten Stelle geht es darum, auf das Heilige zu besinnen. Da dieses das "Höchste" ist, muss das Besinnen darauf "ein höheres sein und einem Denken entstammen, dessen Ursprung selbst dem Heiligen gemäß ist"; dieser Ursprung ist die "trunkene Stirn" (Heidegger, 1982, p. 146). Diese ursprüngliche Trunkenheit, die sich anscheinend auf den "duftenden Becher" in der dritten Strophe des Gedichtes Andenken bezieht (Hölderlin, 1992', p. 361), unterscheidet sich von der "Betrunkenheit" sowie dem " Rausch' der 
Begeisterung", weil sie die "getragene Erhabenheit der Stimmung" für das "nüchtern[e]" Denken an das Heilige ist (Heidegger, 1982, p. 147). Die nüchterne Trunkenheit ist also anders als die berauschende Begeisterung. Demnach ist diese nicht die gleiche Begeisterung, bezüglich derer die Verwandlung in der Feiertagshymne geschieht.

An der zweiten Stelle, an der das Wort "Begeisterung" vorkommt, beschäftigt sich Heidegger mit dem Zusammenhang zwischen Geist und Seele im Hinblick auf die dritte Strophe des Gedichtes Andenken: "[...] Nicht ist es gut,/Seellos von sterblichen/Gedanken zu sein [...]". Heidegger zufolge sind "sterbliche Gedanken" die Gedanken, die den Sterblichen allein gehören, die Hölderlin den "Göttern gegenüber" stellt; "seellos" ist der Mensch, der "auf das Wesenhafte des Menschen" nicht besinnt; dieser Mensch ist "ohne Seele" und kann somit kein "Beseeler" sein (Heidegger, 1982, p. 151). Was Hölderlin mit "Seele" meint, ist für Heidegger (1982, p. 153) das Gemüt, die "Quelle und Stätte des muots - des Mutes in dem ursprünglichen Sinne, daß der Mut Ursprung ist und Innigkeit des Gleichmuts und der Armut, der Sanftmut und des Edelmuts, der Anmut und des Opfermuts, der Großmut und der Langmut". Der Gegensatz zum Seelenlosen ist das "Seelenvolle", der "Mut zum Höchsten". (Heidegger, 1982, p. 153). Die Seele in diesem Sinne steht nicht im Gegensatz zum Geist, sondern dieser ist die "ursprüngliche Erfüllung und die Fülle der Seele" (Heidegger, 1982, p. 154). Dementsprechend wird der Mensch erst mit dem Geist seelenvoll. In diesem Zusammenhang des Geistes mit der Seele (Gemüt) wird folgender Satz verständlich: "In der Begeisterung waltet der ursprüngliche Mut des Gemüts" (Heidegger, 1982, p. 154). Die Begeisterung ist der Zustand, in dem die vom Geist erfüllte Seele herrscht. In diesem Zustand ist der Mensch der Beseeler. Im Beitrag “Andenken” nennt Heidegger den feurigen Geist den "dichtende[n] Geist", der insofern erscheint, als sich seine "dichtenden Gedanken" "in der Seele des Dichters" "sammeln und vollenden" (Heidegger, 1981, p. 91). Wenn die Seele des Dichters "[v]om dichtenden Geist begeistert" wird, ist sie "beseelend" (Heidegger, 1981, p. 91). Der begeisterte Dichter ist also der Beseeler.

Die Rolle dieses Beseelers ist bemerkenswert, und zwar aus zwei Gründen. Zum einen hängt sie damit zusammen, was Heidegger ursprünglich in der Vorlesung Hölderlins Hymne “Andenken” besprechen wollte. Zum anderen erinnert sie uns an die Rolle des Dichters in der Feiertagshymne. Die Rolle des beseelenden Dichters findet sich in folgendem Satz: "Der dichtende Geist gründet durch den Beseeler das dichterische Wohnen der Erdensöhne" (Heidegger, 1981, p. 91). In der oben genannten Notiz, die im September 1941 entstanden ist, listet Heidegger drei Aufgaben der Vorlesung für das Wintersemester 1941/42 auf. Die zweite Aufgabe besteht darin, deutlich zu machen, dass 
die "Erläuterung der beiden ersten Strophen und der Übergang zur dritten" "wesentlich" für die Auslegung des Gedichtes Andenken ist (Heidegger, 1982, p. 197). Für die Ausführung dieser Aufgabe wollte Heidegger auf viele Themen eingehen: "Hölderlins Denken der Geschichte. Die Geschichtlichkeit und das Fest. Das dichterische Wohnen des Menschen. Die Entgegnung der Menschen und Götter. Der Halbgott. Der Dichter. Der freie Gebrauch des Eigenen. Die Gründung im Heimischen. Die Notwendigkeit der Vorbereitung. Das Lernen im 'Gespräch"” (Heidegger, 1982, p. 197). Alle diese Themen außer dem des "dichterische[n] Wohnen[s] des Menschen" werden in der Vorlesung Hölderlins Hymne "Andenken” behandelt. Dieses Thema greift Heidegger, wie gezeigt, erst in seinem Beitrag "Andenken” auf. Um dichterisch zu wohnen, benötigt der Mensch also den dichtenden Geist, der ihm durch die Vermittlung des begeisterten Dichters gegeben wird.

Die vermittelnde Rolle des Dichters erinnert uns an die Feiertagshymne, in der der Dichter das himmlische Feuer ins Lied hüllt und es den Erdensöhnen reicht. Indem sie von diesem gereichten Feuer begeistert werden, wohnen sie dichterisch auf der Erde. Die Dichtung des Dichters geht also ihrem dichterischen Wohnen voraus. Da wir aufgezeigt haben, dass die Verwandlung der Begeisterung bei der Vermittlung des Dichters in der Feiertagshymne geschieht, richten wir unsere Aufmerksamkeit auf die Vermittlung des Dichters im Gedicht Andenken, um die Verwandlung anschaulich zu machen.

Zunächst stellt sich die Frage, wer der Dichter im Gedicht Andenken ist, weil das Wort "Dichter" nur einmal dort vorkommt, und zwar im allerletzten Vers. Heidegger zufolge ist der Dichter der Schiffer. Die Worte "den Schiffern" kommen erstmals in der ersten Strophe des Gedichtes vor, in der der wehende Nordost den Schiffern gute Fahrt über das Meer verheißt. Von dieser Fahrt sagt die fünfte Strophe, die von den Männern spricht, "die am meerbreiten Ausgang des Stromes zu den Indiern gegangen sind", nämlich von den Seefahrern und den Schiffern (Heidegger, 1981, p. 86). Die fünfte und letzte Strophe des Gedichtes endet interessanterweise mit den Worten "die Dichter". Um die Gleichsetzung zwischen den Schiffern und den Dichtern zu begründen, führt Heidegger das Bruchstück Sybille von Hölderlin an: "Der Sturm / die Äste beugt / Und der Rabe singt / So wandert das Wetter Gottes über / Aber du heiliger Gesang / Und suchst armer Schiffer den gewohnten / Zu den Sternen siehe" (Hölderlin, 1916, p. 237; Heidegger, 1981, p. 86). Im Anschluss daran stellt Heidegger (1981, p. 86) fest: "Die Schiffer sind die kommenden Dichter Germaniens."

Darüber hinaus haben die Schiffer und die Dichter bei Hölderlin noch eine grundlegende Gemeinsamkeit. In der Feiertagshymne riskiert der Dichter, "unter Gottes Gewittern" "mit entblößtem Haupte zu stehen”, um das himmlische 
Feuer "mit eigner Hand" zu fassen (Hölderlin, 1992¹, p. 240). Da der Strahl dieses Feuers eine destruktive Wirkung hat, ist der Dichter der tödlichen Gefahr ausgesetzt. Auch im Gedicht Andenken ist der Schiffer derjenige, der ein Risiko eingeht. Er macht die weite Fahrt über das Meer in das fremde Land, in dem er den feurigen Geist erfährt. Der Schiffer kehrt in die Heimat zurück und erzählt seinen Freunden zuhause die Erfahrung des feurigen Geistes. Damit erfahren auch sie diesen Geist, ohne sich der Gefahr ausgesetzt zu haben. Offensichtlich können die Erfahrung des Schiffers in der Fremde und die Erfahrung seiner Freunde in der Heimat bezüglich des feurigen Geistes nicht gleich sein. Die Verwandlung hinsichtlich der Erfahrung ist also geschehen.

Der Schiffer (Dichter) hält sich in der Fremde auf und lernt das Fremde (das Darzustellende), um das Eigene (die Klarheit der Darstellung) frei zu gebrauchen. Dort verschmachtet er wohl vor der Sehnsucht nach der Heimat und ist wegen des feurigen Geistes "fast verbrandt" (Heidegger, 1982, p. 191). Da der Dichter den "inneren Bezug" auf das himmlische Feuer hat, kann er dieses klar darstellen. Mithilfe dieser klaren Darstellung kann er das Heilige "in seinem anfänglichen Kommen" sagen, "ins Wort gründen" und "als Wort den Erdensöhnen" schenken; die "gründende Schenkung des Anfänglichen" ist die Stiftung, weswegen diese Stiftung das Wesen der Dichtung ausmacht (Heidegger, 1982, p. 193). Dieses Wesen hat Hölderlin selbst in den allerletzten Vers des Gedichtes Andenken mit folgenden Worten formuliert: "Was bleibet, aber stiften die Dichter." Im Wesen der Dichtung als Stiftung geschieht also die Verwandlung, damit der feurige Geist ins Wort gegründet und es den Freunden geschenkt wird, so wie das himmlische Feuer ins Lied gehüllt und es den Erdensöhnen gereicht wird.

\section{Schluss}

In der vorliegenden Arbeit haben wir Heideggers Begriff der Verwandlung problematisiert. Der Begriff kommt in seiner Erläuterung des Hölderlinschen Gedichtes Andenken einmal vor und findet keine nähere Erklärung. Da die Verwandlung des Biographischen in das Gedicht beim Dichten geschieht, haben wir uns mit dem Wesen der Dichtung auseinandergesetzt. Dafür wurden Hölderlins Hymne Wie wenn am Feiertage ... und Heideggers Erläuterung dieser Hymne herangezogen. Die Feiertagshymne bringt das Wesen der Dichtung und den Entstehungsprozess des Liedes zum Vorschein. Der Dichter also ahnt die Natur, die als das Heilige und Feurige wahrgenommen wird. Er fasst den feurigen Geist, hüllt ihn ins Lied und gibt es den Erdensöhnen. Das zerstörerische Feuer wird durch den Dichter ins Lied verwandelt, das die Menschen ohne Gefahr 
erfahren können. Auch im Gedicht Andenken haben wir uns die Verwandlung hinsichtlich des himmlischen Feuers aufgezeigt. Der Schiffer (Dichter) fährt über das Meer in die Fremde, in der er das himmlische Feuer erfährt. Er kehrt in die Heimat zurück und erzählt dort den Menschen seine Erfahrung, damit sie es erfahren, ohne sich der Gefahr auszusetzen. Das tödliche Feuer wird durch den Dichter (Schiffer) ins Wort verwandelt und den Erdensöhne geschenkt. Da die Verwandlung eine grundlegende Rolle für die Entstehung der Dichtung spielt, können wir mit Heidegger (1982, p. 30) feststellen, dass der Gedichtinhalt (das Biographische) und das Gedichtete im Gedicht "nicht das Selbe" sind.

\section{Literatur}

“Ausführliches Lateinisch-Deutsches Handwörterbuch” (Band 2). Ed. Heinrich Georges. Hannover: Hahn, 1983.

BAUMANN, E. "Das Geheimnis wird Licht. Friedrich Hölderlin's Gedicht 'Andenken”". Essen: Die Blaue Eule, 1997.

BUßMANN, H. "Lexikon der Sprachwissenschaft". Stuttgart: Alfred Kröner Verlag, 1983.

"Das Bedeutungswörterbuch" (Band 10). Ed. Dudenredaktion. Mannheim/Leipzig/ Wien/Zürich: Duden, 2002.

DERRIDA, J. (1988)."Vom Geist. Heidegger und die Frage”. Frankfurt am Main: Suhrkamp 1992.

FRIEDRICH, J. 'Dichtung als 'Gesang'. Hölderlins 'Wie wenn am Feiertage ...' im Kontext der Schriften zur Philosophie und Poetik 1795-1802”. München: Verlag Wilhelm Fink, 2007.

GEMOLL, W. “Griechisch-Deutsches Schul- und Handwörterbuch”. München/Wien: Freytag, 1988.

HEGEL, G. W. F. "Phänomenologie des Geistes”. Frankfurt am Main: Suhrkamp, 1970. HEIDEGGER, M. "Erläuterungen zu Hölderlins Dichtung”. Frankfurt am Main: Vittorio Klostermann, 1981.

1982.

. "Hölderlins Hymne ‘Andenken’”. Frankfurt am Main: Vittorio Klostermann,

"Herkunftswörterbuch. Etymologie der deutschen Sprache" (Band 7). Ed. Dudenredaktion. Mannheim/Leipzig/Wien/Zürich: Duden, 2001.

HÖLDERLIN, F. “Gedichte 1800-1806” (Hölderlins Sämtliche Werke, Band 4). Ed. Norbert von Hellingrath. München/Leipzig: Müller, 1916.

. "Gedichte nach 1800" (Grosse Stuttgarter Ausgabe, Band 2). Ed. Friedrich

Beißner. Stuttgart: Cotta, 1990.

. "Sämtliche Gedichte". Ed. Jochen Schmidt. Frankfurt am Main: Deutscher Klassiker Verlag, 1992 . 
. "Hyperion/Empedokles/Aufsätze/Übersetzungen". Ed. Jochen Schmidt. Frankfurt am Main: Deutscher Klassiker Verlag, $1992^{2}$.

REUß, R. “... / Die eigene Rede des anderen. Hölderlins Andenken und Mnemosyne”. Frankfurt am Main: Stroemfeld, 1990.

SATTLER, D. "Friedrich Hölderlin. 144 fliegende Briefe" (Band 2). Darmstadt/ Neuwied: Luchterhand, 1981.

SEIFERT, A. “Untersuchungen zu Hölderlins Pindar-Rezeption”. München: Fink, 1982. SZONDI, P. "Hölderlin-Studien mit einem Traktat über philologische Erkenntnis". Frankfurt am Main: Suhrkamp, 1970.

. "Einführung in die literarische Hermeneutik". Frankfurt am Main: Suhrkamp, 1975. 